BBA 72353

\title{
THE INTERACTION OF ADRIAMYCIN WITH CARDIOLIPIN IN MODEL AND RAT LIVER MITOCHONDRIAL MEMBRANES
}

\author{
KLAAS NICOLAY ${ }^{a, *}$, REMY J.M. TIMMERS ${ }^{b}$, ELLEN SPOELSTRA ${ }^{b}$, RONALD VAN DER NEUT ${ }^{b}$, \\ JACO J. FOK $^{b}$, YVONNE M. HUIGEN ${ }^{b}$, ARIE J. VERKLEIJ ${ }^{a}$ and BEN DE KRUIJFF ${ }^{a}$ \\ ${ }^{a}$ Institute of Molecular Biology, and ${ }^{b}$ Department of Biochemistry, State University of Utrecht, Padualaan 8, $3584 \mathrm{CH}$ \\ Utrecht (The Netherlands)
}

(Received June 26th, 1984)

Key words: Antibiotic-membrane interaction; Adriamycin; Cardiolipin; mtRNA; (Rat liver)

The interaction of adriamycin with cardiolipin in model membranes and in various membrane preparations derived from rat liver mitochondria was studied and the results are analyzed in the light of a possible specific interaction between adriamycin and cardiolipin. It was found that adriamycin binds to cardiolipin-containing model membranes with a fixed stoichiometry of two drug molecules per cardiolipin. Furthermore, the extent of drug complexation by mitochondria and mitoplasts (inner membrane plus matrix) is in reasonable agreement with their cardiolipin content. In contrast, adriamycin-binding curves of inner membrane ghosts and submitochondrial particles reveal considerable association to an additional site, presumably RNA. The evidence for the potential importance of RNA as a target comes from experiments on outer membranes and microsomes which both appear to bind substantial amounts of adriamycin. Removal of the major part of the RNA associated with these fractions by EDTA treatment is accompanied by a dramatic reduction of binding capacity. We propose that endogenous RNA present in mitochondria and mitoplasts is not accessible for adriamycin at low concentrations of the drug due to the presence of an intact lipid barrier. This potential site comes to expression in ghosts and submitochondrial particles, due to the absence of an intact lipid bilayer and due to the inside-out orientation of the limiting membrane, respectively. Electron microscopical studies show that adriamycin induces dramatic changes in mitochondrial morphology, similar to the uncoupler-induced effects described by Knoll and Brdiczka (Biochim. Biophys. Acta 733, 102-110 (1983)). Adriamycin has an uncoupling effect on mitochondrial respiration and oxidative phosphorylation. The concentration dependence of this effect correlates with the adriamycin-binding curve for mitochondria which implies that only bound adriamycin actively inhibits respiration.

\section{Introduction}

The anthracycline antibiotic adriamycin is widely accepted as a major chemotherapeutic agent in the treatment of a variety of malignancies [1]. Most of its side effects are also seen with other

\footnotetext{
* To whom correspondence should be addressed. Abbreviations: Hepes, 4-(2-hydroxyethyl)-1-piperazineethanesulphonic acid.
}

anti-neoplastic drugs and are reversible. However, adriamycin-induced cardiotoxicity is very specific and limits the total dose that may be given since the effect is cumulative [2].

At present, no unifying theory is available to explain both the anti-neoplastic and cardiotoxic effects resulting from adriamycin therapy. Nuclear DNA has usually been considered to be the prime target for the anti-neoplastic action [3]. However, this mechanism, based exclusively on the interac- 
tion with DNA and the inhibition of nucleic acid synthesis, has recently been questior,ed. First, adriamycin derivatives having a low affinity towards DNA are still capable of inhibiting cell mitosis [4]. Secondly, Tritton and Yee [5] have demonstrated that adriamycin coupled to an insoluble agarose support is actively cytotoxic towards L1210 cells. No free adriamycin could enter the cells under these conditions, which indicates that the cell surface is a target for the action of the drug.

An even more pronounced role of adriamycinmembrane interactions has recently been suggested to explain the drug-induced cardiomyopathy (for a review, see Ref. 6). The early development of cardiac failure is characterized by changes in the morphology and function of heart mitochondria [7-9]. Moreover, the development of ECG changes shows a good correlation with the impairment of mitochondrial function [8]. Several mechanisms of action have been proposed to explain the adriamycin-induced inhibition of mitochondrial operation, including inhibition of electron transfer and oxidative phosphorylation [10] and initiation of lipid peroxidation [11,12]. The common denominator in these mechanisms is that it involves the action of adriamycin at the mitochondrial membrane level. Moreover, Demant [11] demonstrated that adriamycin-induced lipid peroxidation requires binding of the drug to the inner membrane. Ruysschaert and co-workers [6] have presented evidence strongly suggesting that the formation of a $2: 1$ complex between adriamycin and cardiolipin, a negatively charged phospholipid exclusively localized in the inner mitochondrial membrane, could be the prime cause of the drug toxicity. Recently, Carafoli and coworkers [13] demonstrated that the mitochondrial phosphate carrier, reconstituted in liposomes, is inhibited by adriamycin via complexation of the drug to cardiolipin, the latter being required for enzymatic activity [14].

The present paper deals with the question whether indeed cardiolipin is the prime target for adriamycin in the mitochondrion. The experimental procedure followed to address this problem primarily consists of the determination of the level of adriamycin binding to cardiolipin-containing model membranes and to the various membrane preparations derived from rat liver mitochondria. In addition, the morphological and functional aspects of adriamycin binding to mitochondria have been studied.

\section{Materials and Methods}

Chemicals. Adriamycin (NSC 123127) was a gift from Farmitalia-Carlo Erba (Brussels, Belgium) and the National Cancer Institute. The purity of the adriamycin samples was checked by chromatographic analysis on Silicagel H (Merck, Darmtadt, F.R.G.) using chloroform/methanol/acetic acid/water $(100: 50: 14: 6, \mathrm{v} / \mathrm{v})$ as the developing system. Bovine heart cardiolipin (disodium salt) was obtained from Avanti-Polar Lipids (Birmingham, AL, U.S.A.).

Mitochondria. Mitochondria were isolated from the liver of adult male Wistar rats, essentially according to Vercesi et al. [16] in a medium containing 0.25 M mannitol, $5 \mathrm{mM}$ 4-(2-hydroxyethyl)-1-piperazine-ethanesulphonic acid (Hepes), and $0.5 \mathrm{mM}$ sodium EDTA at pH 7.4. The functional integrity of the final preparation was routinely determined by measuring the respiratory control index [17]. This index was between 4 and 8 when succinate was used as electron donor.

Oxygen consumption by suspensions of mitochondria and inner membrane ghosts was measured polarographically with a Clark-type electrode (Yellow Springs Instruments). A $3.5 \mathrm{ml}$ cell thermostated at $25^{\circ} \mathrm{C}$ was used. The respiratory activities were measured in a buffer containing 130 $\mathrm{mM} \mathrm{KCl}, 3 \mathrm{mM}$ Hepes, $2 \mathrm{mM}$ potassium phosphate, pH 7.4 (buffer A) supplemented with $4 \mathrm{mM}$ potassium succinate and $4 \mu \mathrm{M}$ rotenone. For intact mitochondria, state 3 respiration [17] was initiated by the addition of $300 \mathrm{nmol}$ ADP.

The contamination of the mitochondrial fraction with endoplasmic reticulum and plasma membranes was estimated by measuring the activities of glucose-6-phosphatase [18] and 5'-nucleotidase [19], respectively. Both activities amounted to maximally $2 \%$ of the original activity in the liver homogenate. This extent of contamination compares favourably with data in the literature $[20,21]$.

Preparation and isolation of mitochondrial fractions. The procedures followed for the preparation of mitoplasts (inner membrane plus matrix) was as 
follows [23,24]: (i) mitochondria (approx. $150 \mathrm{mg}$ of protein) were resuspended in $16 \mathrm{ml} 20 \mathrm{mM}$ potassium phosphate ( $\mathrm{pH} 7.4$ ), $0.02 \%$ bovine serum albumin and incubated $10 \mathrm{~min}$ at $0^{\circ} \mathrm{C}$; (ii) after centrifugation $(10 \mathrm{~min}, 12000 \times g)$ the pellet was resuspended in $10.5 \mathrm{ml}$ of the above medium; (iii) subsequently, $3.5 \mathrm{ml}$ of a buffer containing $1.8 \mathrm{M}$ sucrose, $2 \mathrm{mM} \mathrm{ATP}$ and $2 \mathrm{mM} \mathrm{MgSO}_{4}$ was added, and the suspension stirred for $10 \mathrm{~min}$ at $0^{\circ} \mathrm{C}$; (iv) the suspension was sonicated in portions of $3.5 \mathrm{ml}$ for $10 \mathrm{~s}$ at $60 \mathrm{~W}$ with a Branson probe sonifier equipped with a $6 \mathrm{~mm}$ diameter microtip; (v) the mitoplasts were collected by centrifugation (10 $\min , 12000 \times g$ ). The integrity of the mitoplasts and the native orientation of the inner membrane was ascertained by measuring the reduction of ferricyanide, according to Klingenberg [25]. The contamination of the mitoplasts with outer membrane was calculated from the activity of the marker enzyme monoamine oxidase, as described by Weissbach et al. [26]. It appeared that the total activity of monoamine oxidase in the mitoplast fraction varied from 3 to $6 \%$ of the total activity in the original mitochondrial preparation.

Outer membranes [23,24] were isolated from the supernatant of the final mitoplast pellet by centrifugation at $144000 \times g$ for $60 \mathrm{~min}$. Succinate dehydrogenase activity, assayed according to Green et al. [27] was used to determine inner membrane contamination. The total succinate dehydrogenase activity of the outer membrane preparation was approx. $10 \%$ from that of the intact mitochondrial fraction.

Inner membrane ghosts were prepared from mitoplasts as described before [28].

Submitochondrial particles were obtained from mitoplasts by the procedure described by Krebs et al. [29]. The ferricyanide test [25] demonstrated that the orientation of the submitochondrial particle membrane was approx. $80 \%$ inside-out.

Microsomes. Microsomes were isolated from rat liver homogenates as described previously [30]. Ribosomes were detached from the rough microsomes in this preparation by treatment with EDTA as detailed by Godelaine et al. [31].

Lipid preparations. Cardiolipin liposomes were made as described before [15] by vortexing a dry lipid film to a final concentration of approx. 2.5 $\mathrm{mM}$ in buffer $\mathrm{A}$ for $2 \mathrm{~min}$ at room temperature.
Total mitochondrial lipids were extracted from a freshly isolated mitochondrial suspension (10 $\mathrm{ml} ; 15-20 \mathrm{mg}$ of protein $/ \mathrm{ml}$ ), according to Bligh and Dyer [22], except that the water phase contained $50 \mathrm{mM}$ sodium EDTA (pH 7.4). For experiments on binding of adriamycin, a dry lipid film was dispersed to a final concentration of about 10 $\mathrm{mM}$ lipid in buffer $\mathrm{A}$.

On a phosphorus basis, the percentages of the mitochondrial phospholipids were determined to be: cardiolipin $16.2 \%$, phosphatidylethanolamine $36.2 \%$, phosphatidylcholine $42.8 \%$, minor components (mainly phosphatidylinositol and phosphatidylserine) $4.8 \%$.

$X$-ray diffraction. Small angle X-ray diffraction profiles were obtained using a Kratky camera with a $10 \times 0.2 \mathrm{~mm} \mathrm{Cu}-\mathrm{K}_{\alpha}$ beam $(40 \mathrm{kV} ; 20 \mathrm{~mA})$ equipped with a position-sensitive detector (LETI). An entrance slit of $100 \mu \mathrm{m}$ was used. Each measurement took an exposure time of approx. $15 \mathrm{~min}$. Samples were mounted in a temperature-controlled slit $(16 \times 1.5 \times 1.5 \mathrm{~mm})$ between two sheets of cellophane.

Electron microscopy. Freeze-fracture electron microscopy was carried out as described in Ref. 35. For thin sections, specimens were fixed in $2.5 \%$ glutaraldehyde, washed in isotonic phosphate buffer, postfixed with $1 \% \mathrm{OsO}_{4}$, dehydrated with acetone and embedded in araldite. Sections were stained with uranyl acetate and lead citrate and examined in a Philips 301 electron microscope.

Binding of adriamycin to membrane systems. Unless otherwise indicated, appropriate amounts of an adriamycin stock solution $(1.25 \mathrm{mM})$ in buffer A were diluted with buffer $A$ to a final volume of $1.15 \mathrm{ml}$ after which $100 \mu \mathrm{l}$ of freshly prepared membranes was added. After an incubation for 10 min at $25^{\circ} \mathrm{C}$ the samples were centrifuged. The supernatants were diluted with buffer $A$ and measured at $500 \mathrm{~nm}$ with a Perkin-Elmer type 356 double beam spectrophotometer. The concentration of unbound adriamycin was calculated from calibration spectra run with adriamycin solutions of known content in buffer A.

Analytical methods. Phospholipids were quantified by the method of Rouser et al. [32]. Protein was measured according to Peterson [33] with bovine serum albumin as the standard. RNA was determined as described by Hatcher and Goldstein 
[34] with AMP as the standard. Phospholipids were separated by thin-layer chromatography on Kieselgel 60 plates (Merck, Darmstadt, F.R.G.) with chloroform/methanol/acetic acid/water $(90: 40: 12: 2, \mathrm{v} / \mathrm{v})$ as the eluting solvent.

\section{Results}

Adriamycin-cardiolipin interaction in model membranes

Prior to the investigation of adriamycin binding to mitochondrial systems, we have studied its interaction with cardiolipin-containing lipid systems of a well-defined composition. As a start, the binding of adriamycin to pure cardiolipin liposomes was measured (Fig. 1). The strength of the interaction was evident from the visual precipitation of the lipid at the higher adriamycin concentrations. The data demonstrate that saturatable binding occurs. The final level amounts to approx.

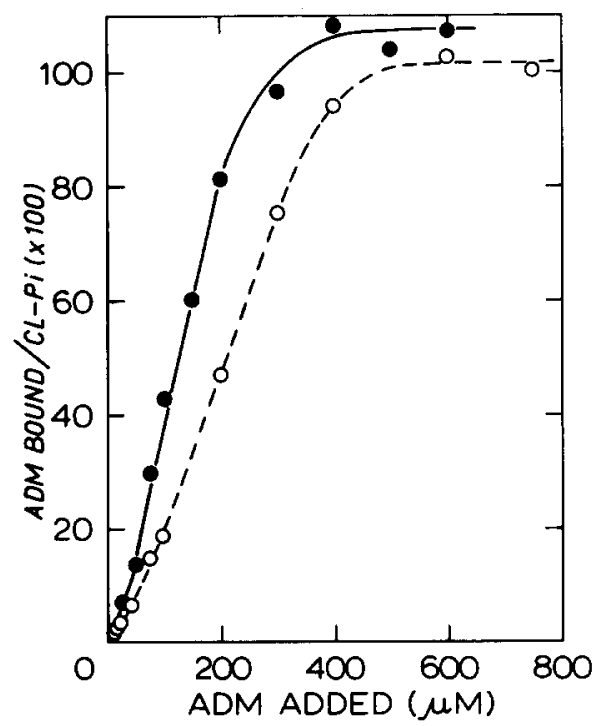

Fig. 1. Binding of adriamycin (ADM) to cardiolipin (CL) liposomes. Two different methods were used for exposing the lipid to adriamycin: (i) adriamycin was added externally after dispersion of $230 \mathrm{nmol}$ cardiolipin in drug-free buffer $(\mathrm{O}-$ -O); (ii) the liposomes were prepared in a buffer containing increasing concentrations of adriamycin (- - ). In the latter method, approx. $250 \mathrm{nmol}$ cardiolipin was dispersed in $1.25 \mathrm{ml}$ adriamycin-containing buffer. Liposomes were sedimented by centrifugation at $30000 \times g$ for $20 \mathrm{~min}$. The amount of adriamycin bound is given as the percentage of the amount of cardiolipin phosphorus present. Other conditions as described in Materials and Methods. one drug molecule per cardiolipin phosphate, i.e. a complex with a stoichiometry of two adriamycin per cardiolipin is formed. Moreover, this stoichiometry is reached both when adriamycin is added externally to preformed liposomes and when adriamycin is included in the buffer used for dispersion of the lipid. It should be noted that the two binding curves depicted in Fig. 1 differ at lower adriamycin concentrations, in that the apparent affinity of adriamycin towards cardiolipin is higher when the drug is present during the formation of the liposomes than when it is added afterwards to preformed liposomes. By plotting the data in Fig. 1 as a function of the free adriamycin concentration it can be estimated that the apparent dissociation constant of the adriamycin-cardiolipin complex is approx. $10 \mu \mathrm{M}$ when the drug is present during formation of the liposomes. However, this number is of limited value since both Scatchard and Hill analysis (not shown) of the data in Fig. 1 indicate that drug binding is of a complex cooperative nature.

In order to investigate whether adriamycin binding to cardiolipin liposomes is accompanied by a structural reorganization when the drug is added externally, we have carried out small angle

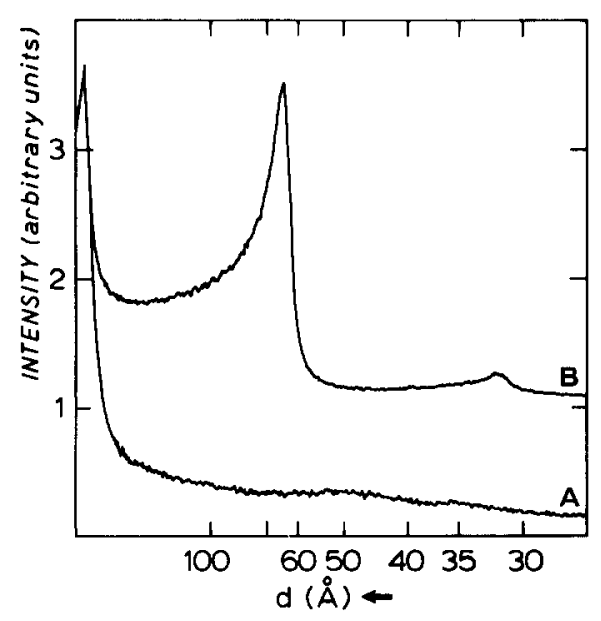

Fig. 2. Small angle $X$-ray diffraction of cardiolipin in the absence (Fig. 2A) and in the presence of adriamycin (Fig. 2B). Cardiolipin liposomes ( $5 \mu \mathrm{mol}$ lipid) were prepared in buffer $A$ as described in Materials and Methods. In Fig. 2B, $15 \mu \mathrm{mol}$ adriamycin in buffer $A$ was added to the suspension. Liposomes were collected by centrifugation for $20 \mathrm{~min}$ at $30000 \times \mathrm{g}$. The experimental conditions used for X-ray diffraction were as given in Materials and Methods. 
$\mathrm{X}$-ray diffraction studies (Fig. 2). It is known from electron microscopy and ${ }^{31} \mathrm{P}-\mathrm{NMR}$ [51] that the disodium salt of cardiolipin forms a lamellar structure upon dispersion in an aqueous buffer. In the pure cardiolipin preparation (Fig. 2A) no discrete diffraction profile is seen. This might be caused by the absence of macroscopic, long range ordering in the system. An alternative explanation would be that the repeat distance is too long to be measured with the camera used. The diffraction pattern changes dramatically upon addition of a saturating amount of adriamycin (Fig. 2B). In this case, first and second order reflections corresponding to a repeat distance of approx. $64 \AA$ are observed which indicates that adriamycin has induced a closely stacked multilamellar ordering in the system.

As a next step, we have investigated the binding of adriamycin to a physiologically relevant mixture of lipids, i.e. the mixture of total rat liver mitochondrial lipids (Fig. 3). As for the pure

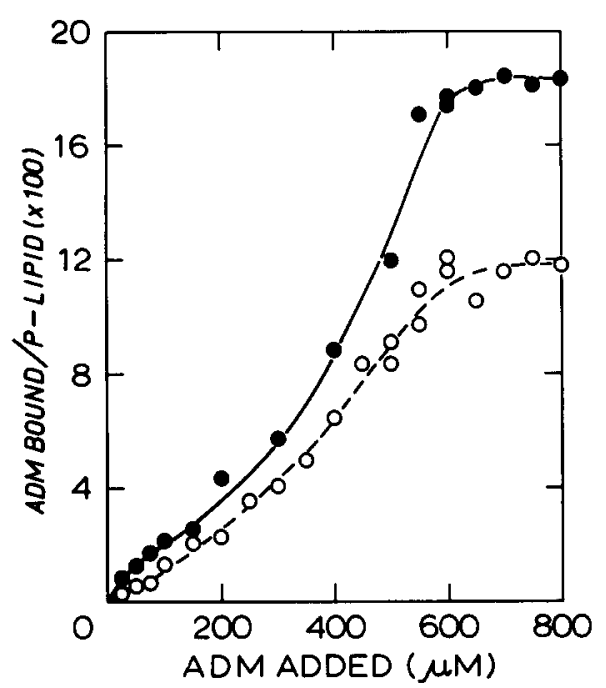

Fig. 3. Binding of adriamycin (ADM) to total rat liver mitochondrial lipids. Lipids were extracted from rat liver mitochondria as described in Materials and Methods. For measuring adriamycin binding, the drug was added either externally to preformed liposomes $(\mathrm{O}--\mathrm{O})$ or liposomes were dispersed in buffer containing increasing concentrations of the drug (- - ). In the latter method, approx. $1 \mu$ mole lipid was dispersed in $1.25 \mathrm{ml}$ adriamycin-containing buffer. Liposomes were sedimented by centrifugation at $30000 \times g$ for $20 \mathrm{~min}$. The amount of bound adriamycin is expressed as the percentage of the amount of lipid phosphorus present. For further details see Materials and Methods. cardiolipin liposomes, drug-lipid interaction is of a complex nature involving cooperative effects. The extent of adriamycin binding amounts to aprox. 18 and $12 \%$ on a lipid phosphorus basis when adriamycin is present during dispersion and when the drug is added externally after dispersion, respectively. The former value is in reasonable agreement with the cardiolipin content of the rat liver mitochondria which amounts to $16.2 \%$ of the phospholipids (see Materials and Methods). This observation shows that arriamycin has access to the complete cardiolipin pool when the drug is present during the formation of the liposomes. In contrast, only approx. $70 \%$ of the cardiolipin is saturated with adriamycin when the latter is added externally. A similar difference between the binding observed for the addition and inclusion method has been reported for the binding of other positively charged agents to cardiolipin and phosphatidylserine liposomes [36].

It is interesting to note that the extent of adriamycin binding to mitochondrial lipid mixtures is significantly lower when the lipids were extracted in the absence of EDTA (data not shown). In this case, the binding levels corresponded to 45 and $22 \%$ of the cardiolipin when the drug was present or absent during the formation of the liposomes, respectively. The difference arising from the extraction procedure most probably is related to the induction of a hexagonal $\mathrm{H}_{\mathrm{II}}$ phase of the cardiolipin by $\mathrm{Ca}^{2+}$ [37] when EDTA is omitted. Cardiolipin when organized in this non-bilayer structure is not accessible for adriamycin (De Kruijff, B., unpublished observations).

It should be noted that the affinity of adriamycin for cardiolipin in the mitochondrial lipid mixture is much lower than for pure cardiolipin (Fig. 1). The apparent dissociation constant of the adriamycin-cardiolipin complex in the former case is in the order of $300 \mu \mathrm{M}$.

In conclusion, adriamycin binds to cardiolipin in model membranes with a stoichiometry of $2: 1$. This stoichiometry is preserved upon binding of the drug to the mixture of mitochondrial lipids.

Binding of adriamycin to mitochondrial membranes

As pointed out in the Introduction, the present study primarily addresses the question whether 
cardiolipin is the major site of interaction for adriamycin in mitochondria. To tackle this problem, we have measured the binding of adriamycin to intact rat liver mitochondria (Fig. 4A). Up to drug concentrations of $250 \mu \mathrm{M}$, there are two major adriamycin binding sites in the intact mitochondrion: (i) a site corresponding to approx. $5 \mathrm{nmol}$ adriamycin bound/mg of protein, which is saturated around $40 \mu \mathrm{M}$ adriamycin; (ii) another site which, upon saturation around $200 \mu \mathrm{M}$
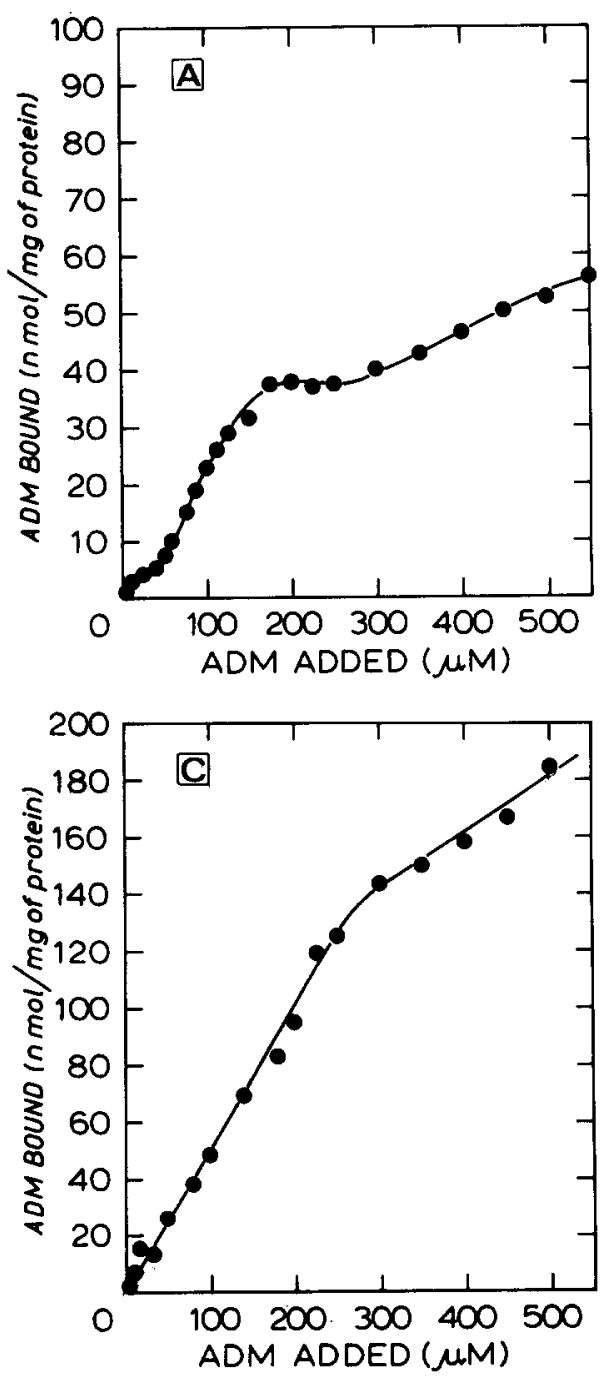

adriamycin, gives rise to a final level of approx. 37 $\mathrm{nmol}$ adriamycin bound/mg of protein. It is worth stressing that Fig. 4A represents an equilibrium situation since identical curves were obtained using incubation times with drug of 5,10 and $30 \mathrm{~min}$ (data not shown). The binding of adriamycin by mitoplasts (inner membrane plus matrix) is also characterized by the presence of two saturatable binding sites, which give equal contributions to the final level of approx. $26 \mathrm{nmol}$ adriamycin
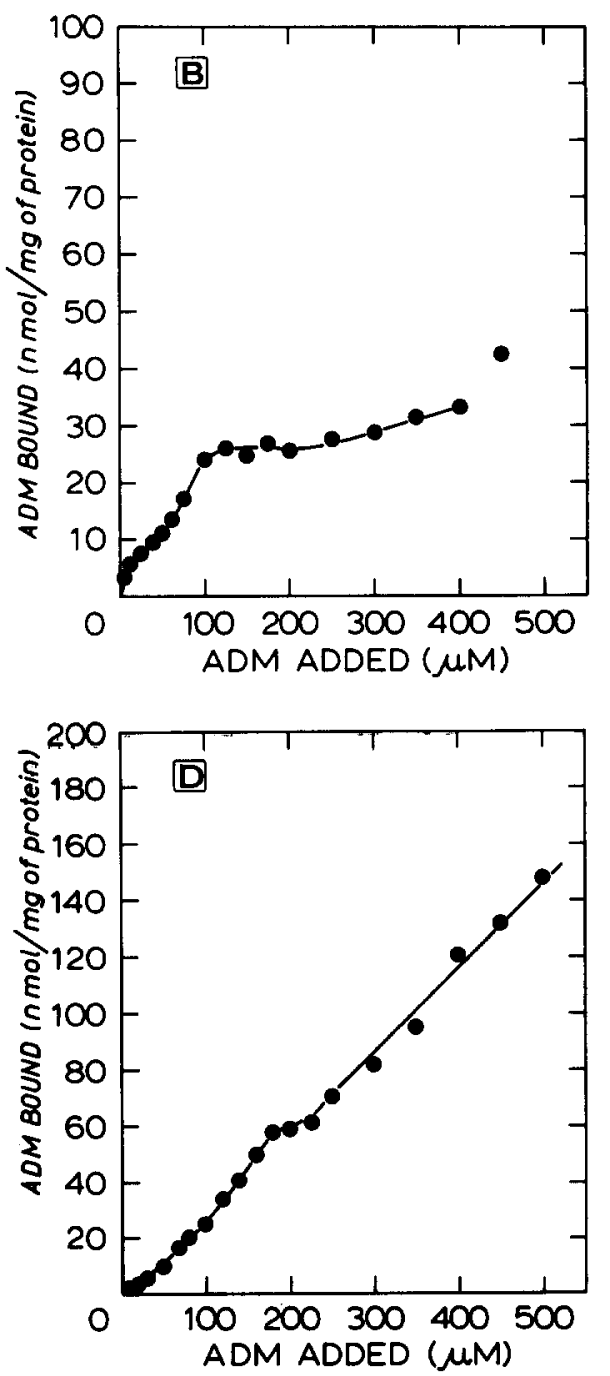

Fig. 4. Binding of adriamycin (ADM) to intact rat liver mitochondria (A), mitoplasts (B), inner membrane ghosts (C) and submitochondrial particles (D). The amounts of protein used at each adriamycin concentration were $2.0,1.25,0.5$ and $1.0 \mathrm{mg}$ for Fig. $4 A, B, C$ and D, respectively. Mitochondria and mitoplasts were sedimented by centrifugation at $12000 \times g$ for 10 min. Ghosts were sedimented at $30000 \times \mathrm{g}$ for $15 \mathrm{~min}$ and submitochondrial particles at $150000 \times \mathrm{g}$ for $30 \mathrm{~min}$. For further details see Materials and Methods. 
bound/mg of protein (Fig. 4B). By comparing Figs. $4 \mathrm{~A}$ and $4 \mathrm{~B}$, it can be concluded that, although there is significant adsorption to the outer membrane (see also below), the major mitochondrial binding site for adriamycin resides in the mitoplast. This is in agreement with the proposed role of cardiolipin in binding since this lipid is exclusively found in the inner membrane. Moreover, there is reasonable agreement between the cardiolipin content of mitochondria and mitoplasts (33 and $32 \mathrm{nmol}$ cardiolipin phosphorus/mg of protein, respectively) and the levels of constant binding observed in Figs. 4A and 4B, respectively.

At adriamycin concentrations above $300 \mu \mathrm{M}$ low affinity sites become accessible in both the mitochondrial and mitoplast preparations (Figs. 4A and 4B). This phenomenon shows up even more pronounced in adriamycin binding curves for inner membrane ghosts (Fig. 4C) and submitochondrial particles (Fig. 4D). As a consequence, discrete levels of specific binding are apparently absent in these systems. However, it should be noted that ghosts and submitochondrial particles show a significantly increased binding relative to mitochondria and mitoplasts which is to be expected on the basis of the increased cardiolipin content of the former preparations (65 and 88 nmol cardiolipin phosphorus/mg of protein for ghosts and submitochondrial particles, respectively).

Some indications for the possible origin of the described differences between mitochondria and mitoplasts on one hand and ghosts and submitochondrial particles on the other hand came from adriamycin binding studies to outer mitochondrial membranes (Fig. 5A) and microsomes (Fig. 5B). Fig. 5A shows that, on a protein basis, the outer mitochondrial fraction binds considerable amounts of adriamycin corresponding to approx. $140 \mathrm{nmol}$ adriamycin $/ \mathrm{mg}$ of protein. When the relative contribution of outer membrane protein to total mitochondrial protein is considered, we estimate that in the intact mitochondrion, 5-7 nmol adriamycin/mg of mitochondrial protein is associated with the outer membrane. However, the above consideration is hampered by the fact that our outer membrane preparation is contaminated with inner membrane fragments (see Materials and Methods). Consequently, it also contains cardiolipin amounting to approx. $46 \mathrm{nmol}$

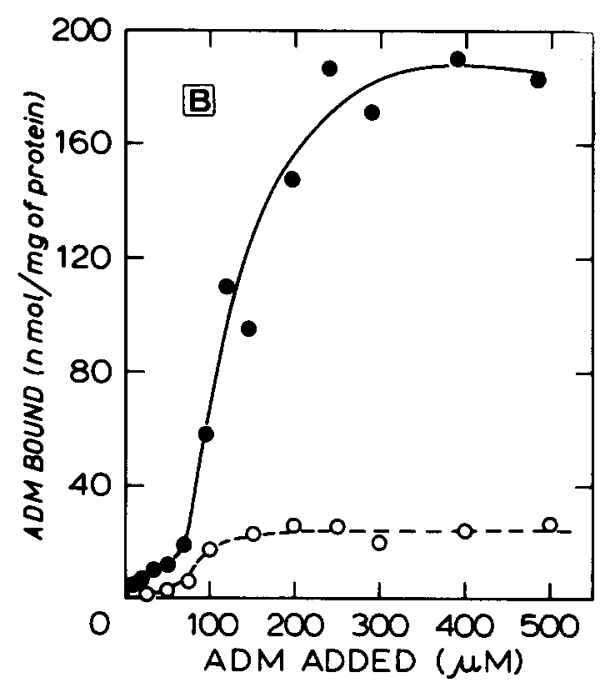

Fig. 5. Binding of adriamycin (ADM) to outer mitochondrial membranes (A) and microsomes (B). Outer membranes and microsomes were treated with EDTA to strip off the membrane-associated ribosomes, as described in Materials and Methods. The amounts of protein used were 0.5 and $0.25 \mathrm{mg}$ for native (-_) and stripped outer membranes $\left(\mathrm{O}_{-}-\mathrm{O}_{-}\right.$), respectively, and 0.4 and 0.3 $\mathrm{mg}$ for native (-) and stripped microsomes $\left(\mathrm{O}_{-}-\mathrm{O}\right)$, respectively. Microsomes were sedimented as described before [25]; outer membranes were sedimented by centrifugation for $15 \mathrm{~min}$ at $30000 \times \mathrm{g}$. The average RNA contents were determined to be 73 and $45 \mathrm{nmol}$ nucleotide/ $\mathrm{mg}$ of protein for native and stripped outer membranes, respectively, and 165 and $87 \mathrm{nmol} \mathrm{nucleotide} / \mathrm{mg}$ of protein for native and stripped microsomes, respectively. For further details see Materials and Methods. 
cardiolipin phosphorus/mg of outer membrane protein. However, the extent of adriamycin binding observed greatly exceeds this number (Fig. 5A). Therefore, there must be additional sites to which substantial adriamycin binding can occur.

Besides for cardiolipin, adriamycin is known to have a high affinity for binding to DNA and RNA [38]. The rough endoplasmic reticulum membrane which represents the major source of non-nuclear RNA is known to be partially continuous with the outer mitochondrial membrane [39]. Therefore, fragments of the endoplasmic reticulum membrane might remain associated with the outer mitochondrial membranes upon isolation. The validity of this suggestion is confirmed by the fact that significant RNA levels were detected in the outer membrane preparation (see the legend to Fig. 5). To test whether RNA associated with the endoplasmic reticulum can bind considerable amounts of adriamycin, we have carried out two sets of experiments. First, adriamycin binding to freshly isolated microsomes was measured. Secondly, ribosomes associated with the rough microsomal fraction in the pool of total microsomes were removed by EDTA treatment, whereafter adriamycin binding to these stripped microsomes was determined. Fig. 5B depicts the results of the above experiments. Whereas there is substantial adriamycin complexation by native microsomes, binding by stripped microsomes is dramatically reduced. It is important to note that the stripping process is primarily accompanied by a loss of ribosomal RNA and protein while phospholipid is completely conserved (data not shown). Therefore, it is concluded that, in microsomes, RNA represents the major binding site for adriamycin.

Subsequently, the effect of stripping the outer membrane fraction on its ability to bind adriamycin was measured (Fig. 5A). As for the microsomes, loss of RNA is accompanied by a considerable reduction in drug binding while the phospholipid content, and thus the amount of cardiolipin is not influenced (not shown).

\section{Effect of adriamycin on mitochondrial morphology}

In view of the interaction of adriamycin with the inner mitochondrial membrane lipid, cardiolipin, it was of interest to study the effects of the drug on the overall structure and morphology of mitochondria in vitro. To this aim we have performed electron microscopy studies on rat liver mitochondria. Figs. $6 \mathrm{a}$ and $6 \mathrm{c}$ show thin sections and freeze-fracture electron micrographs of control mitochondria, respectively. Fig. 6 a exhibits the well-known condensed configuration of freshly isolated liver mitochondria [41]. Control mitochondria frozen from $25^{\circ} \mathrm{C}$ contain a random distribution of intramembranous particles embedded in a smooth continuum of the hydrophobic interior of both limiting membranes, as revealed by Fig. 6c.

The dramatic effects of adriamycin on overall morphology are most clearly seen in thin sections (Fig. 6b). The originally condensed configuration (Fig. 6a) has changed into a structure in which the inner membrane is pressed closely to the outer limiting membrane (Fig. 6b), while cristae are not longer visible. These data demonstrate that on a morphological basis the inner membrane is affected most. It should be noted that the total mitochondrial volume does no appear to be affected by adriamycin. In contrast, preliminary turbidity measurements suggest that adriamycin induces a concentration-dependent swelling of resting mitochondria; the swelling becomes even more pronounced during respiration, in excess of the well-known energy-dependent swelling of mitochondria (not shown). Adriamycin-induced swelling is a well-known aspect of mitochondrial impairment observed in vivo [2]. Freeze-fracture micrographs reveal that, in the presence of adriamycin, the fracture faces through both the inner and outer membrane remain randomly covered with protein particles (Fig. 6d). Thus, adriamycin-cardiolipin complex formation does not cause an isothermal lateral phase separation into protein-free and protein-rich regions of the

Fig. 6. Electron micrographs of rat liver mitochondria collected from buffer $\mathrm{A}(\mathrm{a}, \mathrm{c})$ or from buffer $\mathrm{A}$ supplemented with $500 \mu \mathrm{M}$ adriamycin $(\mathrm{b}, \mathrm{d})(80000 \times)$. After isolation, mitochondria $(2 \mathrm{mg}$ of protein $/ \mathrm{ml})$ were incubated for $10 \mathrm{~min}$ at $25^{\circ} \mathrm{C}$ in the relevant media. Subsequently, mitochondria were concentrated by a brief centrifugation. In Fig. $6 \mathrm{c}$ patches (arrow) of the outer membrane are seen on top of the inner membrane. For further details see Materials and methods. 

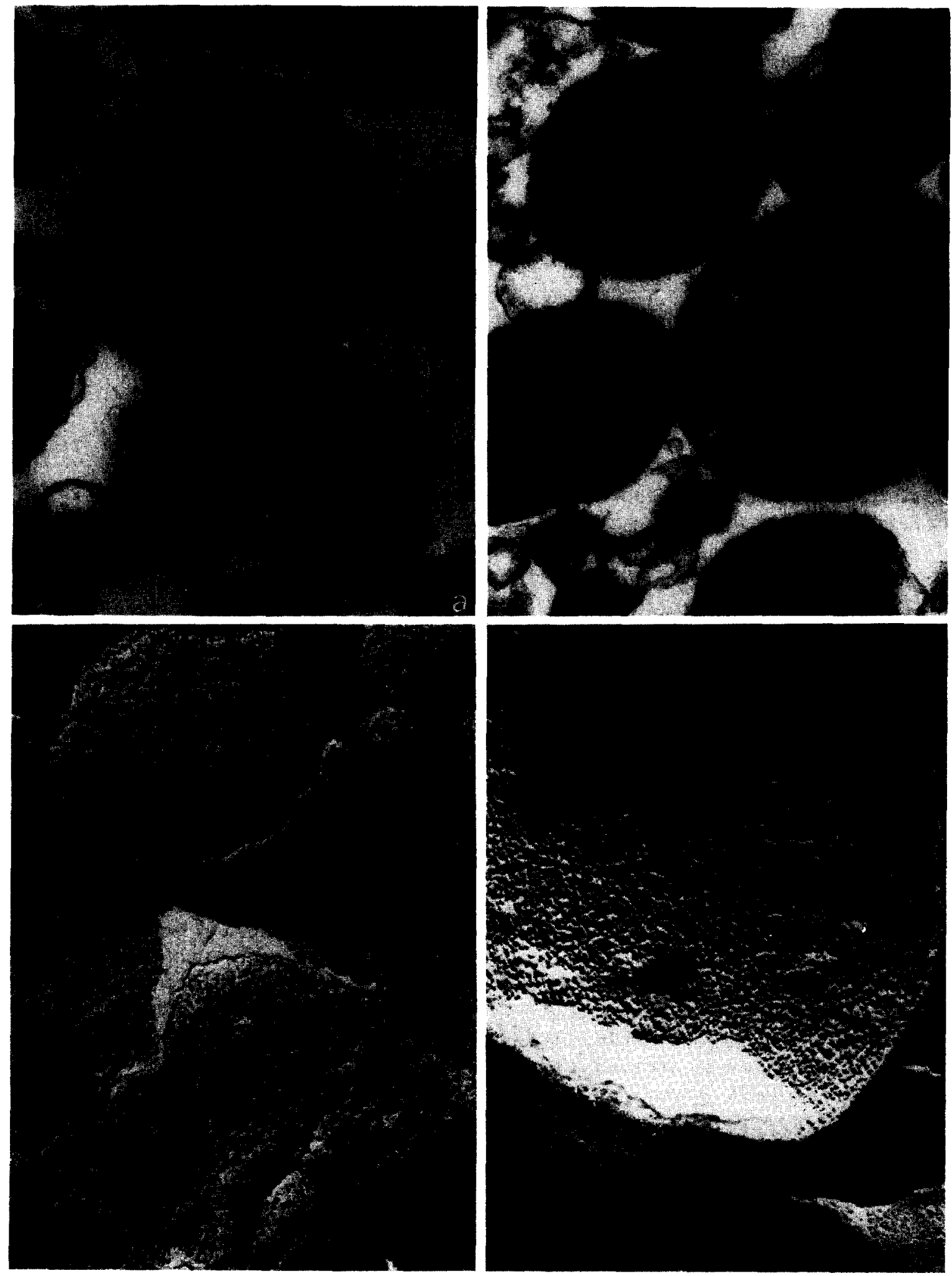
inner mitochondrial membrane, which is observed upon cooling [41].

Freeze-fracture micrographs also reveal another interesting aspect of the action of adriamycin. Control mitochondria exhibit pathwork-like structures (Fig. 6c), which have been interpreted as being the consequence of contact sites between the inner and outer limiting membranes [35,42]. Recently, Brdiczka and co-workers [42] have correlated the frequency of the intermembrane jumping with the metabolic state of the mitochondria. This frequency is highest under phosphorylating conditions, whereas all contact sites are abolished by uncoupling agents. The latter phenomenon was also observed when freeze-fractured mitochondria treated with adriamycin were examined (Fig. 6d). Patchwork-like structures were essentially absent. This observation is most probably relevant for the bioenergetic effects exerted by adriamycin which will be discussed next.

\section{Effects of adriamycin on respiration and oxidative phosphorylation}

Adriamycin is known to influence respiration of mitochondria from normal [43] and tumor cells [44]. In an attempt to correlate the binding pattern of adriamycin to its functional effects, a dose-response curve for rat liver mitochondrial respiration and oxidative phosphorylation was measured (Fig. 7). It can be seen that, in the presence of increasing concentrations of adriamycin, respiration becomes gradually uncoupled from oxidative phosphorylation. The uncoupling action, which is most evident from the respiratory control index, starts around $80 \mu \mathrm{M}$ and has come to completion at approx. $200 \mu \mathrm{M}$ adriamycin. It is interesting to note that whereas state 4 respiration is initially stimulated by adriamycin, state 3 respiration is only inhibited. This demonstrates that the drug is more potent under conditions of oxidative phosphorylation, in agreement with recent reports by Muhammed et al. [10,43]. Whereas respiratory control is very sensitive to adriamycin, the $P / O$ ratio is hardly affected (not shown), in agreement with data in the literature [8].

Apart from respiration by intact mitochondria, also oxygen consumption by inner membrane ghosts and the effect of adriamycin on this process was measured (data not shown). Respiration by

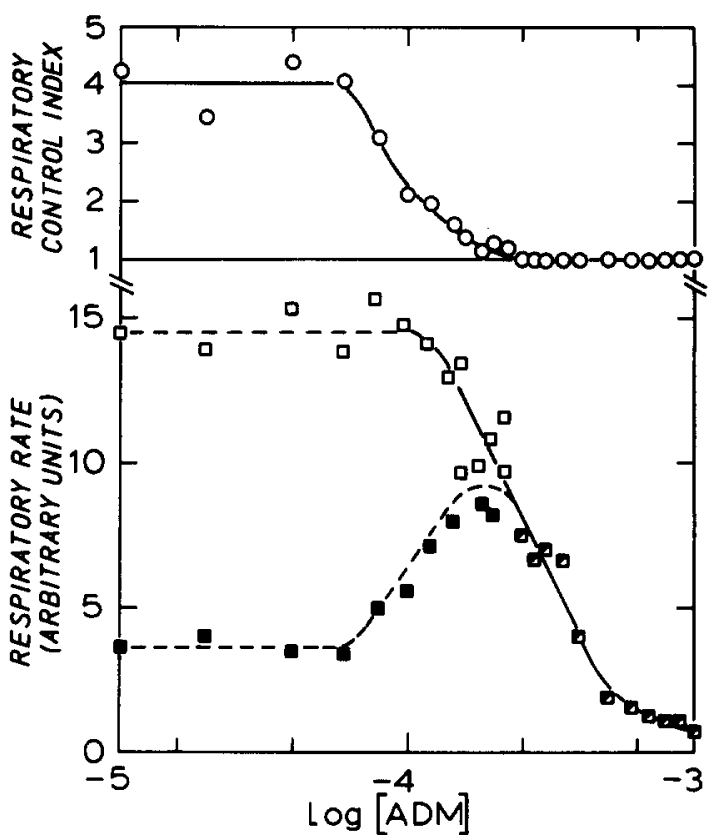

Fig. 7. Effect of adriamycin (ADM) on respiratory control in rat liver mitochondria. Freshly isolated mitochondria $1 \mathrm{mg}$ of protein) were used according to the procedure described in Materials and Methods. Respiratory rates are given in the presence of ADP (state 3; 0 ) and on exhaustion of the added ADP (state $4 ; \boldsymbol{w}$ ). Respiratory control index $(O)$ is defined as the ratio of state 3 and state 4 respiration. Further details are given in Materials and Methods.

ghosts which is completely uncoupled due to the absence of a membrane barrier towards protons, was gradually inhibited by increasing concentrations of adriamycin and became essentially zero around $250 \mu \mathrm{M}$.

It is important to stress that the effects of adriamycin on respiration of both mitochondria and ghosts were almost instantaneous, i.e. respiratory rates were essentially linear throughout the concentration range used. This means that adriamycin can easily reach the inner membrane and thus, that the outer membrane is leaky towards the drug.

\section{Discussion}

The present paper demonstrates that in protein-free model membranes the anti-tumor drug adriamycin forms a stoichiometric complex with the negatively charged phospholipid, cardiolipin. 
The observed stoichiometry, i.e. two adriamycin per cardiolipin, is in agreement with the monolayer data published by Ryusschaert and coworkers [45]. These authors explained the high affinity of adriamycin for cardiolipin by two essential interactions: (i) an electrostatic interaction between the protonated amino group of the sugar residue of adriamycin and the two negatively charged phosphates of cardiolipin; (ii) a ringstacking interaction between adjacent anthraquinone chromophores [6].

As for the model membranes, also the level of adriamycin binding to mitochondria and mitoplasts (Figs. 4A and 4B) was in reasonable agreement with the total cardiolipin content of these preparations. This conclusion still holds if the contribution of the outer membrane in binding to mitochondria is taken into account. Cardiolipin is known to be asymmetrically distributed across the inner mitochondrial membrane [29] such that the main part of the lipid is in the inner monolayer. Since the outer membrane does not represent a barrier to the drug, adriamycin has direct access to cardiolipin in the outer monolayer of the inner membrane, whereas it has to pass the latter membrane in order to reach the major fraction of the lipid which is localized in the inner leaflet. On the basis of these considerations, it is tempting to speculate that the two levels of specific binding observed in the binding curves for mitochondria and mitoplasts (Figs. 4A and $4 B$, respectively) are the consequence of a difference in apparent affinity of the drug for cardiolipin in the outer and the inner monolayer. However, this interpretation does not hold in quantitative terms since the distribution of cardiolipin would change from an asymmetric one for mitochondria (Fig. 4A) to an almost symmetric one for mitoplasts (Fig. 4B). This possibility can be ruled out since the limiting membrane of the mitoplasts had retained its original sidedness and integrity as evidenced by appropriate enzymatic tests (see Materials and Methods). Therefore, we favour the explanation that the complex binding pattern of adriamycin by inner membrane cardiolipin is due to complex cooperative phenomena which are also observed upon adriamycin binding to the mixture of mitochondrial lipids (Fig. 3).

Our data show that apart from cardiolipin also
RNA should be considered as a potential target for adriamycin binding by mitochondria. This could be concluded from the observation that the partial removal of ribosomal RNA from the microsomal and outer membrane fractions led to a dramatic reduction in adriamycin binding capacity (Fig. 5). In addition, rat liver mitochondria, and consequently mitoplasts, contain considerable amounts of RNA, up to $25 \mathrm{nmol}$ nucleotide $/ \mathrm{mg}$ of protein [40]. In contrast, DNA can be excluded from the present discussion since rat liver mitochondrial DNA levels are only in the order of $2 \mathrm{nmol}$ nucleotide/mg of protein [40]. This amount of DNA would only correspond to $0.4 \mathrm{nmol}$ adriamycin bound/mg of protein [46] when all mitochondrial DNA would be saturated with adriamycin.

As detailed above, the level of binding of adriamycin to mitochondria and mitoplasts was in reasonable agreement with the total cardiolipin content of these preparations. If, indeed, cardiolipin is the essentially exclusive target for adriamycin in mitochondria and mitoplasts (at least up to $300 \mu \mathrm{M}$ adriamycin), there must be a special reason why the matrix RNA represents only a low affinity binding site for adriamycin. The presence of an intact limiting membrane might well play a role in this context. This is suggested by the adriamycin binding curve for inner membrane ghosts (Fig. 4C). This curve shows that two sets of binding sites are accessible over the entire concentration range. It should be noted that ghosts still contain considerable amounts of RNA (in the order of $50 \mathrm{nmol}$ RNA nucleotide/mg of protein). Our interpretation of these data is that, in ghosts, adriamycin not only binds to cardiolipin but also to RNA. The latter compound is easily reached by adriamycin, since the ghost membrane no longer offers a barrier towards the drug. The binding of adriamycin to submitochondrial particles could then be explained in similar terms. The latter fraction has approx. $30 \mathrm{nmol}$ RNA nucleotides $/ \mathrm{mg}$ of protein associated with its limiting membrane. This membrane, however, is oriented inside-out for about $80 \%$. Therefore, externally added adriamycin has free access to membrane-associated RNA which would explain why the levels of bound adriamycin substantially exceed the cardiolipin content of the particles. A more 
quantitative description of the adriamycin-RNA interaction will have to await binding experiments of adriamycin to isolated mitochondrial RNA species.

A comparison of the adriamycin-binding curve to mitochondria (Fig. 4A) and the adriamycin concentration dependence of mitochondrial respiration (Fig. 7) demonstrates that the progression of uncoupling of respiration from oxidative phosphorylation parallels the progressive saturation of the higher affinity binding sites. This is in agreement with observations made by others that adriamycin can only affect respiratory activities $[11,47]$ when it is bound to the inner mitochondrial membrane. Moreover, Demant [11] demonstrated that the inhibition of cytochrome $c$ oxidase by adriamycin is the consequence of adriamycin-lipid interactions rather than direct drug-enzyme interactions. This also makes cardiolipin a likely target for adriamycin causing inhibition of the terminal oxidase since this enzyme has an essential requirement for cardiolipin $[48,49]$. In addition, Goormaghtigh et al. [37] have demonstrated that adriamycin can effectively displace both reduced and oxidized cytochrome $c$ from cardiolipin liposomes wich might also play a role in the druginduced inhibition of cytochrome $c$ oxidase activity.

The reason for the (initial) stimulation of state 4 respiration might be 2 -fold: (i) bound adriamycin has been shown to participate in respiration between complex I and complex III [47,50]; (ii) binding of adriamycin might increase the passive proton permeability of the inner membrane, i.e. act as an uncoupler. We favour the latter explanation since it is difficult to visualize stimulation of state 4 respiration under conditions of intact respiratory control. Moreover, the freeze-fracture studies (Fig. 6) reveal similar effects for adriamycin as have been reported to be caused by uncouplers [42], in that contact sites between the inner and outer mitochondrial membranes are abolished. Van Venetie and Verkleij [35] have proposed a semi-fusion model for the contact sites in which non-bilayer lipids are involved. If this model is correct, adriamycin would certainly interfere with the formation of contact sites since the drug blocks the ability of cardiolipin to adopt non-bilayer structures [37].
In view of the data presented in the present paper, considering the binding of adriamycin to mitochondria and the effects it has on mitochondrial respiration, oxidative phophorylation and morphology, we conclude that cardiolipin represents the major target of the drug in these organelles. Therefore, we propose that the mitochondrial deformations which are among the earliest signs of the development of cardiomyopathy during adriamycin therapy [7-9], are the consequence of the strong and specific interaction of the drug with inner mitochondrial membrane cardiolipin.

\section{Acknowledgements}

We wish to gratefully acknowledge the contribution of Mrs. J. Leunissen-Bijvelt in performing the electron microscopy studies. The authors are grateful to Farmitalia and the National Cancer Institute for providing adriamycin. This research was supported by a grant from the Netherlands Organization for the Fight against Cancer (Koningin Wilhelmina Fonds).

\section{References}

1 Young, R.C., Ozols, R.F. and Myers, C.E. (1981) N. Engl. J. Med. 305, 139-153

2 Solcia, E., Ballerini, L., Bellini, O., Magrini, U., Bertazzolli, C., Tosana, G., Sala, L., Balconi, F. and Rallo, F. (1981) Tumori 67, 461-472

3 Simpkins, H., Pearlman, L.F. and Thompson, L.M. (1984) Cancer Res. 44, 613-618

4 Israel, M., Modest, E.J. and Frei, E. (1975) Cancer Res. 35, 1365-1368

5 Tritton, T.R. and Yee, G. (1982) Science 217, 248-250

6 Goormaghtigh, E. and Ruysschaert, J.M. (1984) Biochim. Biophys. Acta 779, 271-288

7 Arena, E., Arico, M., Biondo, F., D'Allessandro, N., Dusonchet, L., Gebbia, N., Gerbasi, F., Sanguedolce, R. and Rausa, L. (1975) Adriamycin Review, Part II, 160-172

8 Bachmann, E., Weber, E. and Zbinden, G. (1975) Agents Actions 5, 383-393

9 Porta, E.A., John, N.S., Matsumura, L., Nakasone, B. and Sablau, H. (1983) Res. Commun. Chem. Pathol. Pharmacol. 41, 125-137

10 Muhammed, H., Ramasarma, T. and Kurup, C.K.R. (1982) Biochim. Biophys. Acta 722, 43-50

11 Demant, E.J.F. (1983) Eur. J. Biochem. 137, 113-118

12 Demant, E.J.F. and Jensen, P.K. (1983) Eur. J. Biochem. $132,551-556$

13 Cheneval, D., Muller, M. and Carafoli, E. (1983) FEBS Lett. 159, 123-126 
14 Kadenbach, B., Mende, P., Kolbe, H.V.J., Stipani, I. and Palmieri, F. (1982) FEBS Lett. 139, 109-112

15 Cullis, P.R. and De Kruijf, B. (1978) Biochim. Biophys. Acta 507, 207-218

16 Vercesi, A., Reynafarje, B. and Lehninger, A.L. (1978) J. Biol. Chem. 253, 6379-6385

17 Chance, B. and Williams, G.R. (1955) J. Biol. Chem. 217, 409-427

18 Swanson, M.A. (1955) Methods Enzymol. 2, 541-543

19 Aronson, N.N. and Tonster, O. (1974) Methods Enzymol. 41, 90-102

20 Reinhart, P.H., Taylor, W.M. and Bygrave, F.L. (1982) Biochem. J. 204, 731-735

21 Kurup, C.K.R., Aithal, H.N. and Ramasarma, T. (1970) Biochem. J. 116, 773-779

22 Bligh, E.G. and Dyer, W.J. (1959) Can. J. Biochem. Physiol. 37, 911-918

23 Parsons, D.F. and Williams, G.R. (1967) Methods Enzymol. $10,443-448$

24 Sottocasa, G.L., Kuylenstierna, B., Ernster, L. and Bergstrand, A. (1967) Methods Enzymol. 10, 448-463

25 Klingenberg, M. (1979) Methods Enzymol. 56, 229-233

26 Weissbach, H., Smith, T.E., Daly, J.W., Witkop, B. and Udenfriend, S. (1960) J. Biol. Chem. 235, 1160-1163

27 Green, D.E., Mii, S. and Kohout, P.M. (1955) J. Biol. Chem. 217, 551-567

28 Cullis, P.R., De Kruijff, B., Hope, M.J., Nayar, R., Rietveld, A. and Verkleij, A.J. (1980) Biochim. Biophys. Acta $600,625-635$

29 Krebs, J.J.R., Hauser, H. and Carafoli, E. (1979) J. Biol. Chem. 254, 5308-5316

30 Van den Besselaar, A.M.H.P., De Kruijff, B., Van den Bosch, H. and Van Deenen, L.L.M. (1978) Biochim. Biophys. Acta 510, 242-255

31 Godelaine, D., Beaufay, H., Wibo, M. and Ravoet, A.-M. (1983) J. Cell Biol. 97, 340-350

32 Rouser, G., Fleischer, S. and Yamamoto, A. (1970) Lipids 5, 494-496
33 Peterson, G.L. (1977) Anal. Biochem. 83, 346-356

34 Hatcher, D.W. and Goldstein, G. (1969) Anal. Biochem. 31, 42-50

35 Van Venetie, R. and Verkleij, A.J. (1982) Biochim. Biophys. Acta 692, 397-405

36 Rietveld, A., Sijens, P., Verkleij, A.J. and De Kruijff, B. (1983) EMBO J. 2, 907-913

37 Goormaghtigh, E., Vandenbranden, M., Ruysschaert, J.M. and De Kruijff, B. (1982) Biochim. Biophys. Acta 685, 137-143

38 Simpkins, H., Pearlman, L.F. and Thompson, L.M. (1984) Cancer Res. 44, 613-618

39 Morre, D.J., Kartenbeck, J. and Franke, W.W. (1979) Biochim. Biophys. Acta 559, 71-152

40 Nass, S., Nass, M.K. and Hennix, U. (1965) Biochim. Biophys. Acta 96, 426-435

41 Hackenbrock, C.R., Hochli, M. and Chau, R.M. (1976) Biochim. Biophys. Acta 455, 466-484

42 Knoll, G. and Brdiczka, D. (1983) Biochim. Biophys. Acta $733,102-110$

43 Muhammed, H. and Kurup, C.K.R. (1984) Biochem. J. 217, 493-498

44 Mailer, K. and Petering, D.H. (1976) Biochem. Pharmacol. 25, 2085-2089

45 Goormaghtigh, E., Chatelain, P., Caspers, J. and Ruysschaert, J.M. (1980) Biochim. Biophys. Acta 597, 1-14

46 Zunino, F., Di Marco, A., Zaccara, A. and Gambetta, R.A. (1980) Biochim. Biophys. Acta 607, 206-214

47 Goormaghtigh, E., Pollakis, G. and Ruysschaert, J.M. (1983) Biochem. Pharmacol. 32, 889-893

48 Fry, M. and Green, D.E. (1980) Biochem. Biophys. Res. Commun. 93, 1238-1246

49 Goormaghtigh, E., Brasseur, R. and Ruysschaert, J.M. (1982) Biochem. Biophys. Res. Commun. 104, 314-320

50 Davies, K.J.A., Doroshow, J.H. and Hochstein, P. (1983) FEBS Lett. 153, 227-230

51 De Kruijff, B. and Cullis, P.R. (1980) Biochim. Biophys. Acta 602, 477-490 